\title{
UNIQUE DETERMINATION OF STRUCTURE AND VELOCITY BY 3-D TOMOGRAPHIC INVERSION OF REFLECTED AND REFRACTED WAVES
}

\author{
Tilahun Mammo \\ Department of Earth Sciences, Faculty of Science, Addis Ababa University \\ PO Box 1176, Addis Ababa, Ethiopia. E-mail: tmammo@geol.aau.edu.et
}

\begin{abstract}
Accurate determinations of interface depths and velocity fields are crucial for the oil industries in their search for hydrocarbon reservoirs. However, the so called velocity-depth ambiguity has always been a problem with the seismic reflection data that is normally used in petroleum exploration. In this paper the cause of this velocity-depth ambiguity is examined and a methodology is proposed that minimizes non-uniqueness in the inversion results. It is shown that simultaneous inversion of zero offset and offset reflection data as well as refraction data can reproduce accurate velocity-depth model using only certain picked seismic events. A subsequent 2-D Prestack depth migration based on the Kirchhoff method utilizing the velocity field obtained from the tomographic inversion extracts more information from the data and gives a clear picture of the subsurface. The superiority of the simultaneous inversion of the reflected and refracted waves to that of reflected waves alone is demonstrated using real data.
\end{abstract}

Key words/phrases: Inversion, non-uniqueness, pre-stack, tomography, velocity-depth ambiguity

\section{INTRODUCTION}

Normal-incidence (zero-offset) reflection data have been extensively used by petroleum industries in hydrocarbon exploration. From these data geophysicists attempt to map the subsurface and delineate oil bearing horizons from the waves recorded on the various sensors systematically deployed on the ground surface in general. They attempt to map the depths to the various layers traversed by the waves and determine their velocity values by analyzing the time of arrivals of the waves. Various studies, however, have demonstrated that the velocity and depth information cannot be uniquely retrieved from the arrival times. The traveltime information cannot be separated into velocity and depth components (Bickel, 1990; Stork and Clayton, 1991; Lines, 1993; Ross, 1994; Tieman, 1994) and consequently a clear picture of the subsurface cannot be obtained. It is, therefore, necessary to understand when and under what conditions the velocity-depth ambiguity occurs and propose a solution whereby these two parameters are uniquely determined thus avoiding the non-uniqueness problem. The principal sources of non-uniqueness in traveltime tomography can be grouped into four:

1. Discrete parameterization of the initial model. In the tomographic inversion the continuous medium we want to image by the seismic waves is discretized into cells or pixels the sizes of which are subjective and left to the individual's choice. This cell parameterization should be consistent with the data to be analyzed to avoid non-uniqueness. Cell size is data dependent (Berryman, 1990).

2. Picking errors in traveltime data. During initial phase of data analysis due to incorrect picking of the arrival times errors may be introduced into the solution of the tomographic problem. Noise and data incoherency are some of the factors that often determine the size of the picking errors. These errors propagate into the final results and greatly affect the model produced (Lines, 1993; Schuster, 1989).

3. Band limitation of the seismic data. Acquired seismic data are always band limited. The seismic data, due to recording instrument limitation and attenuation of the seismic waves during propagation, typically have frequency bandwidth that falls in the range of 5-60 HZ. This band limitation, however, has serious consequences. It is clear that the missing high frequencies affect the resolution of the inversion results (Carrion, 1987). But absence of low frequencies also causes serious problems-the inverse problem becomes highly unstable and the velocity-depth ambiguity is introduced in the inversion results. 
4. Incomplete angular coverage. In medical tomography, complete data coverage is achieved from all angles by rotating the sources and receivers around a patient to map the abnormal tissue. In applied seismology the recording system has limited length with sources placed either at the earth's surface or in boreholes. The data thus collected have limited angular coverage and the seismic waves illuminate only a small portion of the target one desires to map. The situation is aggravated for the zero offset reflection seismic where angular coverage is practically zero. The tomographic images thus obtained from a non-adequate ray angular coverage are not clear, they are blurred.

The problem associated with the discrete parameterization may be handled by trying different parameterization for the same data until consistent results are obtained and the results become insensitive to the parameterization (Berryman, 1990).

Measurement errors in traveltimes of reflected waves have been known to lead to highly ambiguous results. A large traveltime pick error in one layer can affect the velocity estimate in all layers (Schuster, 1989). Picking is generally accurate when done in the post-stack domain as a great deal of the propagation events is removed and reflection events are clearly identified. In prestack domain the situation is not so smooth. Various propagation effects like diffractions and multiply reflected events obscure the identification and picking of the primary events. Picking of refracted waves, on the other hand, gives accurate results as the waves are the first to arrive at the recording sensors and are not contaminated or obscured by other events. This will serve as a quality control in picking and will be shown with examples how important this is to eliminate ambiguities. It should also be remembered that the inversion method one chooses should be robust enough to accommodate small errors in traveltime pickings.

Band limitation and limited angular coverage render the seismic data incomplete. They cause the seismic inversion problem to be ill-posed and lead to numerical instability, non-uniqueness of inversion results or nonexistence of a solution (Carrion and Kuo, 1984). The real problem in seismic tomography is thus to reconstruct a complete picture of the subsurface using incomplete data.

In this paper it is shown how simultaneous inversion of large- and short-offset data in prestack domain can handle the above problems and produce improved resolution of the tomographic imaging. In the inversion process it is a common practice to independently analyze zero-offset and large-offset reflection data as well as refraction arrivals. However, in this paper it is shown that the accuracy and resolution of the seismic parameters are enhanced and the velocity-depth ambiguities are resolved if tomographic inversion of all these data are performed simultaneously.

\section{PROBLEM FORMULATION AND LINEARIZATION}

The practice of discretizing the medium through which the seismic wave propagates into long pixels (cells) between the interfaces is followed in this work. In addition, the continuous interface depth is also discretized into a number of segments (White, 1989). A simple two-layer earth model is considered to analyze the traveltime along a ray path for both reflected and refracted arrivals.

\section{Reflected waves}

The traveltime curve for the geometry given in Figure 1 is

$$
T_{R}=\frac{2 Z_{1} S_{1}}{\cos \theta_{1}}, \quad \cos \theta_{1}=\frac{Z_{1}}{\left(Z_{1}^{2}+X^{2}\right)^{1 / 2}}
$$

where $S_{1}$ is the slowness (reciprocal of velocity) of the medium and $X$ is the half offset. This equation represents a nonlinear relationship between the traveltime $T_{R}$ and depth $Z_{1}$.

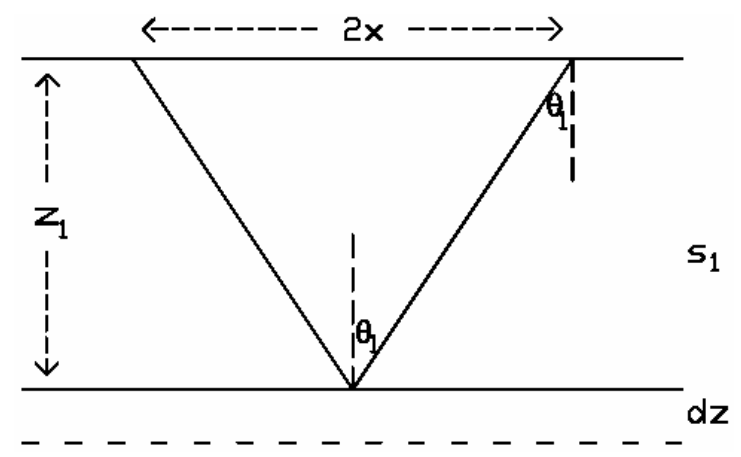

Fig. 1. Reflected ray geometry and reflector depth perturbation in a double layered velocity model.

The problem can be linearized by choosing a reference model with a background parameters of $S_{0}$ and $Z_{0}$ and perturb this reference medium to minimize the difference between measured and computed traveltimes. The slowness perturbation 
$\delta s_{1}$ and interface depth perturbation $\delta z_{1}$ are given by

$$
\begin{aligned}
& \delta s_{1}=S_{1}-S_{0} \\
& \delta z_{1}=Z_{1}-Z_{0}
\end{aligned}
$$

The corresponding perturbation of the traveltime is given by differentiating equation 1 with respect to slowness and interface depth.

$$
\delta \mathrm{t}_{\mathrm{R}}=\frac{2 \mathrm{Z}_{1}}{\cos \theta_{1}} \delta s_{1}+2 \mathrm{~S}_{1} \cos \theta_{1} \delta \mathrm{z}_{1}
$$

For a set of $\mathrm{M}$ rays, a linear system of equations formed from (3) is given by

$$
\delta t_{R}=A^{R} \delta s+B^{R} \delta z
$$

\section{REFRACTED WAVES}

The geometry of the refracted waves is given by the simple model in Figure 2.

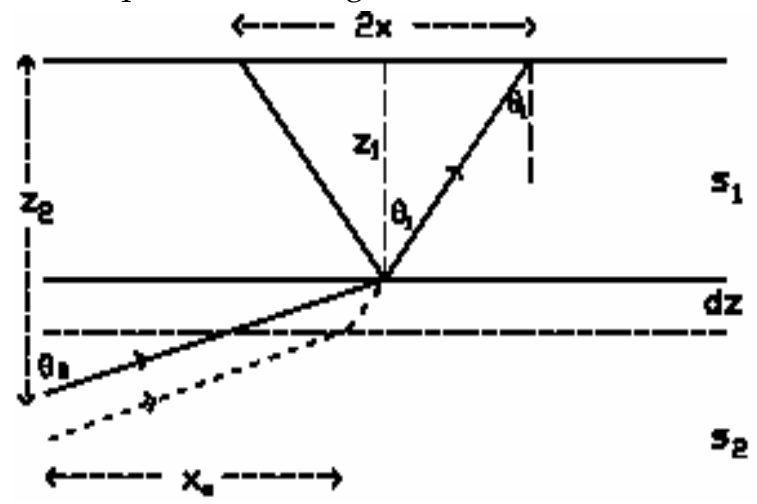

Fig. 2. Refracted ray geometry and refractor depth perturbation in a double layered velocity model.

The traveltime for this wave is given by

$$
\operatorname{Tr}=\frac{Z_{1} S_{1}}{\cos \theta_{1}}+\frac{\left(Z_{2}-Z_{1}\right) S_{2}}{\cos \theta_{2}}
$$

where $\cos \theta_{1}=\frac{Z_{1}}{\left(Z_{1}^{2}+\left(2 X-X_{o}\right)^{2}\right)^{1 / 2}}$,

$$
\cos \theta_{2}=\frac{Z_{2}-Z_{1}}{\left(\left(Z_{2}-Z_{1}\right)^{2}+X_{0}^{2}\right)^{1 / 2}}
$$

The perturbed traveltime is given by

$$
\begin{aligned}
\delta t_{r}= & \frac{Z_{1}}{\cos \theta_{1}} \delta s_{1}+\frac{Z_{2}-Z_{1}}{\cos \theta_{2}} \delta s_{2} \\
& +\left(S_{1} \cos \theta_{1}-S_{2} \cos \theta_{2}\right) \delta z_{1}
\end{aligned}
$$

For a set of $\mathrm{M}$ rays equation 6 can be written as

$$
\delta \mathbf{t}_{\mathrm{r}}=\mathbf{A}^{\mathrm{r}} \delta \mathbf{s}+\mathbf{B}^{\mathrm{r}} \delta \mathbf{z}
$$

Equations 4 and 7 can be combined to give a general matrix representation for both reflected and refracted waves.

$$
\delta \mathbf{t}=\mathbf{A} \delta \mathbf{s}+\mathbf{B} \delta \mathbf{z}
$$

\section{VELOCITY-DEPTH AMBIGUITY}

The relationship between offsets and velocitydepth ambiguity can be examined considering equations 3 and 6 . From these equations it is clear that the effects of the two perturbations are controlled by the cosine terms. The effect of the reflector depth is proportional to the cosine of the angle the seismic wave makes with the vertical whereas that of the slowness is proportional to the inverse of the cosine of this angle. It is to be noted that for small angle $\theta$, in other words for small offset, the difference between $(1 / \cos \theta)$ and $\cos \theta$ is small and consequently the two perturbations are indistinguishable (Stork, 1992; Ross, 1994; Tieman, 1994; Mao and Stuart, 1997). For $\theta$ is equal to zero (zero offset) the two terms are equal. This in essence is the velocity-depth ambiguity. A sufficiently large $\theta$ (large offset) is needed to estimate the effect of the individual perturbation. In the case of refracted waves (equation 6) additional parameters associated with $\cos \theta_{2}$ term contribute to the resulting traveltime perturbation. Even in this case equation 6 suggests that for zero offset the velocity and depth perturbations are completely ambiguous.

In the following illustration equation 3 is used to quantify the dependence of the velocity-depth ambiguity on the offset distance. Assuming an interface depth of $2100 \mathrm{~m}$ and a velocity of 2500 $\mathrm{m} / \mathrm{s}$ above this interface the two-way time is determined to be 1.68 second at zero offset. Let us also assume that the two-way traveltime has now become 1.63 second after model perturbation. This corresponds to a traveltime anomaly of $-50 \mathrm{~ms}$. The problem that needs addressing is how much of this anomaly is caused by a depth perturbation and how much is due to a slowness perturbation. Figure 3 shows the traveltime curves versus offsetto-depth ratio for three situations, namely, when there is no traveltime perturbation and when there is traveltime perturbation that is entirely due to either depth or slowness perturbation. The effect of perturbations is clearly seen from the behavior of the curves. The problem is to differentiate between the two perturbations. At zero offset-to-depth ratio, that is at zero offset, the two perturbations are identical. One cannot separate the effect of one from the other and it is not possible to determine whether the observed traveltime perturbation is due to the interface depth perturbation or due to the slowness (reciprocal of velocity) perturbation. Hence the velocity-depth ambiguity. However, the two perturbations become separate and distinct as 
the offset-to-depth ratio increases, which is for large offset data. Figure 4 shows the two perturbations with the background traveltime subtracted out. With an increase in offset the difference between the two perturbations increases. Taking into account the typical time- picking accuracy of $4-8 \mathrm{~ms}$, it can be seen from the figure that the minimum offset-to-depth ratio at which the effect of the two perturbations become distinct is 1 , where a difference of about $11 \mathrm{~ms}$ is observed. For the ratio of less than 1 the two effects are indistinguishable.

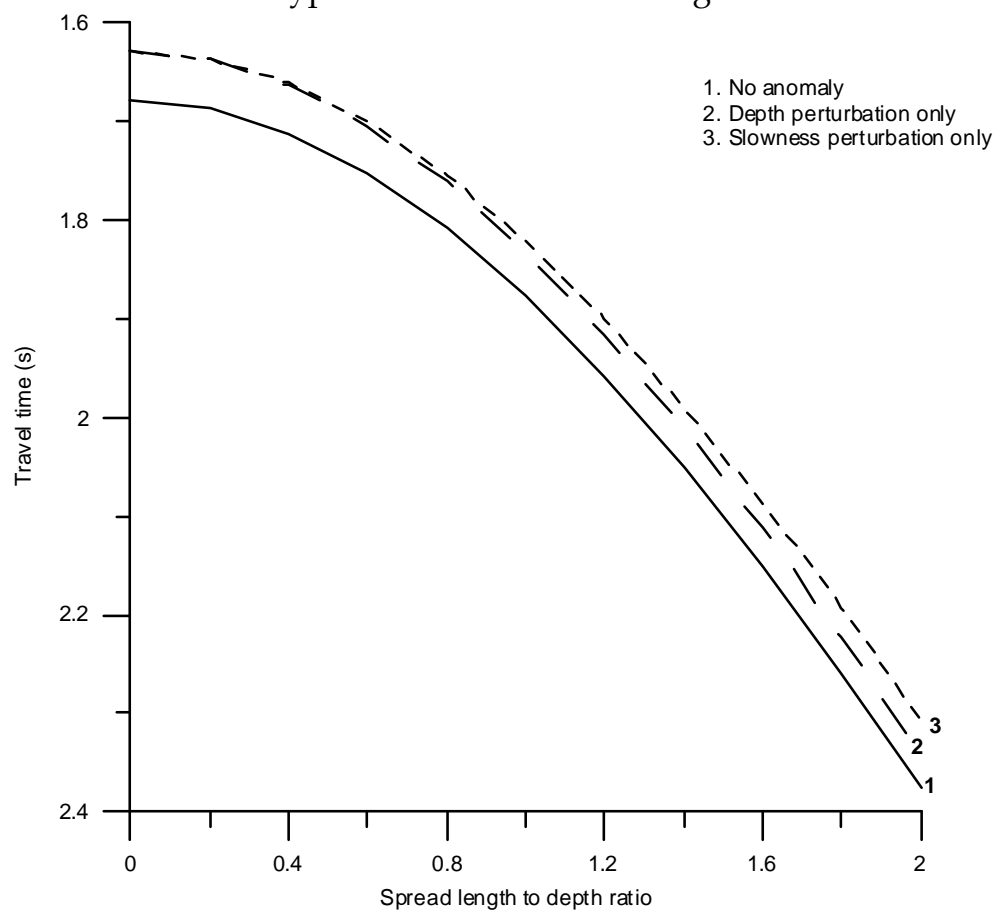

Fig. 3. Traveltime curves with no perturbation, with slowness-only perturbation, and with depth-only perturbation.

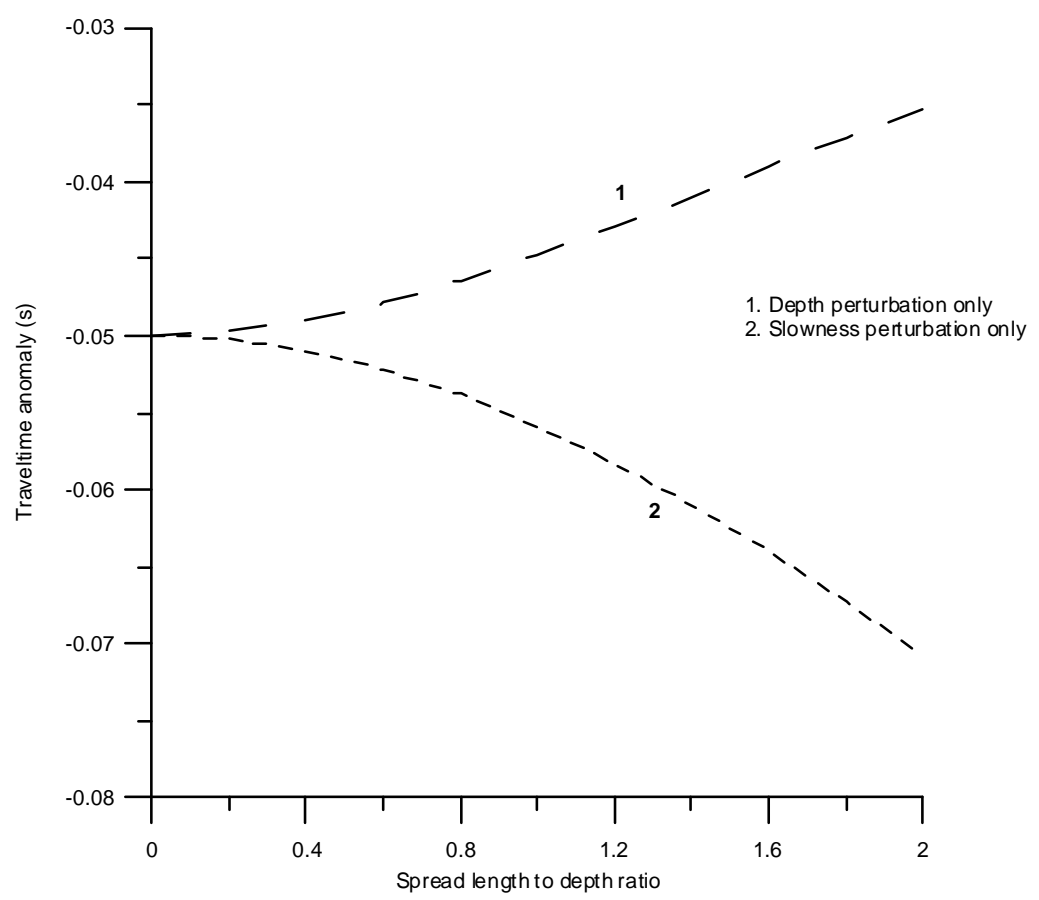

Fig. 4. Traveltime anomaly for slowness-only and depth-only perturbations. 
This case clearly demonstrates that the zero offset reflection stacked data used in petroleum exploration with the usual common-depth-point (CDP) technique fail to resolve the velocity-depth ambiguity. For optimum interface depth and velocity imaging an offset-to-depth ratio of greater than 1 should be used in field data acquisition and processing should be carried out in the pre-stack domain. Pre-stack data contain wide range of offsets and therefore introduce into the inversion procedure the angle $\theta$ that plays an important role in the reconstruction of the velocity and depth functions.

From the above analysis it has become evident that when the angle $\theta$ is zero, as in the case in zerooffset seismic section, it is almost impossible to invert for the velocity and depth separately. It is, therefore, necessary that seismic data with a wide range of offsets (refraction / offset or wide-angle reflection) be used in conjunction with the zerooffset data to resolve the non-uniqueness of inversion results and improve the structural image and velocities of the derived earth model.

\section{TOMOGRAPHIC INVERSION}

Simultaneous inversions of zero offset reflection, large offset reflection and refraction data were performed by solving equation 8 as reproduced here

$$
\delta \mathbf{t}\left(\mathrm{x}_{\mathrm{s}}, \mathrm{x}_{\mathrm{r}}\right)=\mathbf{A} \delta \mathrm{s}+\mathbf{B} \delta \mathrm{z}
$$

where $\mathrm{x}_{\mathrm{S}}$ and $\mathrm{x}_{\mathrm{r}}$ are the coordinates of sources and receivers. $\mathbf{A}$ and $\mathbf{B}$ are tomographic matrices that map the traveltime residuals $\delta \mathbf{t}$ into slowness $\delta \mathbf{s}$ and depth $\delta \mathbf{z}$. This equation is typically underdetermined. That is, the number of rays covering the model space is much smaller than the number of cells of discretization. Thus the matrix A has a null space and we have infinite solution instead of a unique solution. On the other hand, the matrix $B$ is the diagonal matrix of the angles of propagation and inclinations of the interface at the point of reflection. As such it is free from null space (Calnan and Schuster, 1989; Bohm, et al., 1993).

Towards solving equation 9 Carrion et al., (1993) expressed it as

$\delta \mathbf{t}\left(\mathrm{x}_{\mathrm{s}}, \mathrm{x}_{\mathrm{r}}\right)=\left\{\left(\gamma / \lambda^{\mathrm{T}} \mathbf{B B}^{\mathrm{T}} \lambda\right)^{1 / 2} \mathbf{B} \mathbf{B}^{\mathrm{T}} \lambda\right\}+\mathbf{A} \mathbf{A}^{\mathrm{T}} \lambda$

where $\lambda$ is the vector of Lagrangian multipliers and the constraint $\gamma$ is a scalar that prevents large variations of the reflection / refraction points with respect to the expected interface.

$$
\delta \mathbf{z}^{\mathrm{T}} \delta \mathbf{z} \leq \gamma
$$

Solving equation 10 with respect to $\lambda$, we separately recover slowness and depth perturbations

$$
\begin{aligned}
& \delta \mathbf{s}=\mathbf{A}^{\mathrm{T}} \lambda \\
& \delta \mathbf{z}=\left\{\left(\gamma / \lambda^{\mathrm{T}} \mathbf{B B}^{\mathrm{T}} \lambda\right)^{1 / 2}\right\} \mathbf{B}^{\mathrm{T}} \lambda
\end{aligned}
$$

But we see that $\lambda$ depends on $\gamma$ suggesting the need for additional condition - the minimization of variance of the reflection/refraction points about the model interface.

$$
\operatorname{var}(z)=E\left(z^{c}-E\left(z^{i}\right)\right)^{2}
$$

where $z^{C}$ stands for the computed points and $z^{\mathrm{i}}$ represents the initial model which is updated in the course of iterations. By minimizing this variance together with the traveltime residuals it is possible to find the slowness value which provides the smallest values of the variance. In other words, the variance attains the minimum value only for a reasonable velocity estimate.

It is clear that the vector $\mathbf{A}^{\mathrm{T}} \lambda$ in equation 12 is always perpendicular to any vector $\xi$ belonging to the null space of $\mathbf{A}$ and hence the influence of the null space is completely eliminated and any combination of the vectors belonging to the null space is unable to influence the slowness result obtained. $\delta$ s and $\xi$ are independent. However, incomplete angular coverage in model space due to the limited length of the recording system produced a blurred image (Carrion et al., 1992).

\section{SIMULTANEOUS TOMOGRAPHIC INVERSION OF SEISMIC DATA}

The data set processed was a 3D marine prospect acquired in the North Sea along 10 parallel profiles with a ship towing two streamers about $100 \mathrm{~m}$. apart (Johnstad et al., 1993; 1995). Data were collected along 120 channels per streamer with the offsets between the source and receivers ranging from 80 to $3050 \mathrm{~m}$. Both the receiver (hydrophones) and shot intervals are $25 \mathrm{~m}$. each. The total number of shots for most line was about 500 . The first step of processing consists of picking the traveltimes of the refracted waves and prominent reflected events. The direct waves are also included in the picking. The major events that correlate along the 
whole survey are usually identified in the common offset sections and the pickings were performed afterwards in the pre-stack domain. Figure 5 shows the zero offset stacked section. A few picked reflected/refracted events in the common shot gather are shown in Figure 6.

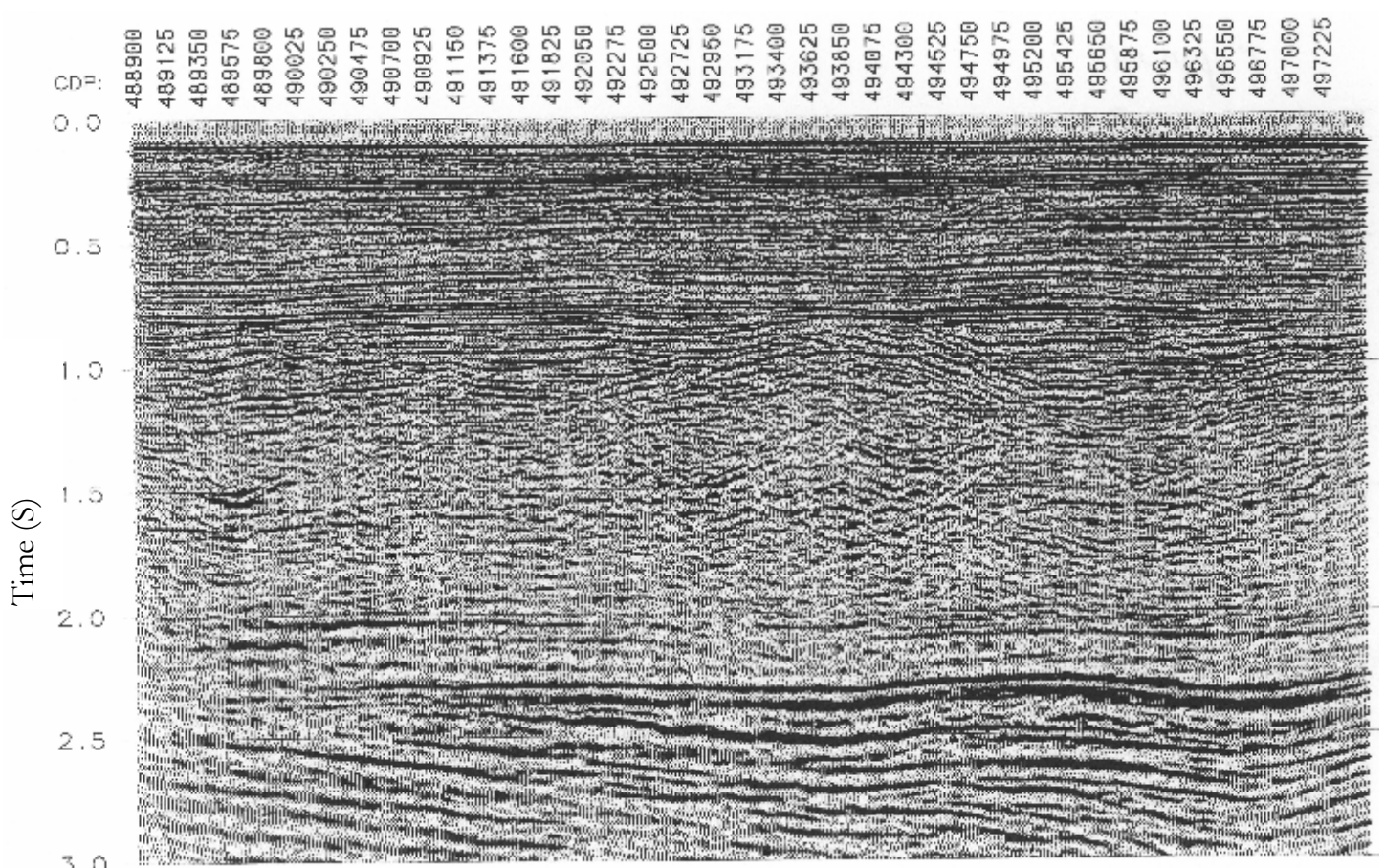

Fig. 5. Zero offset stacked reflection data.

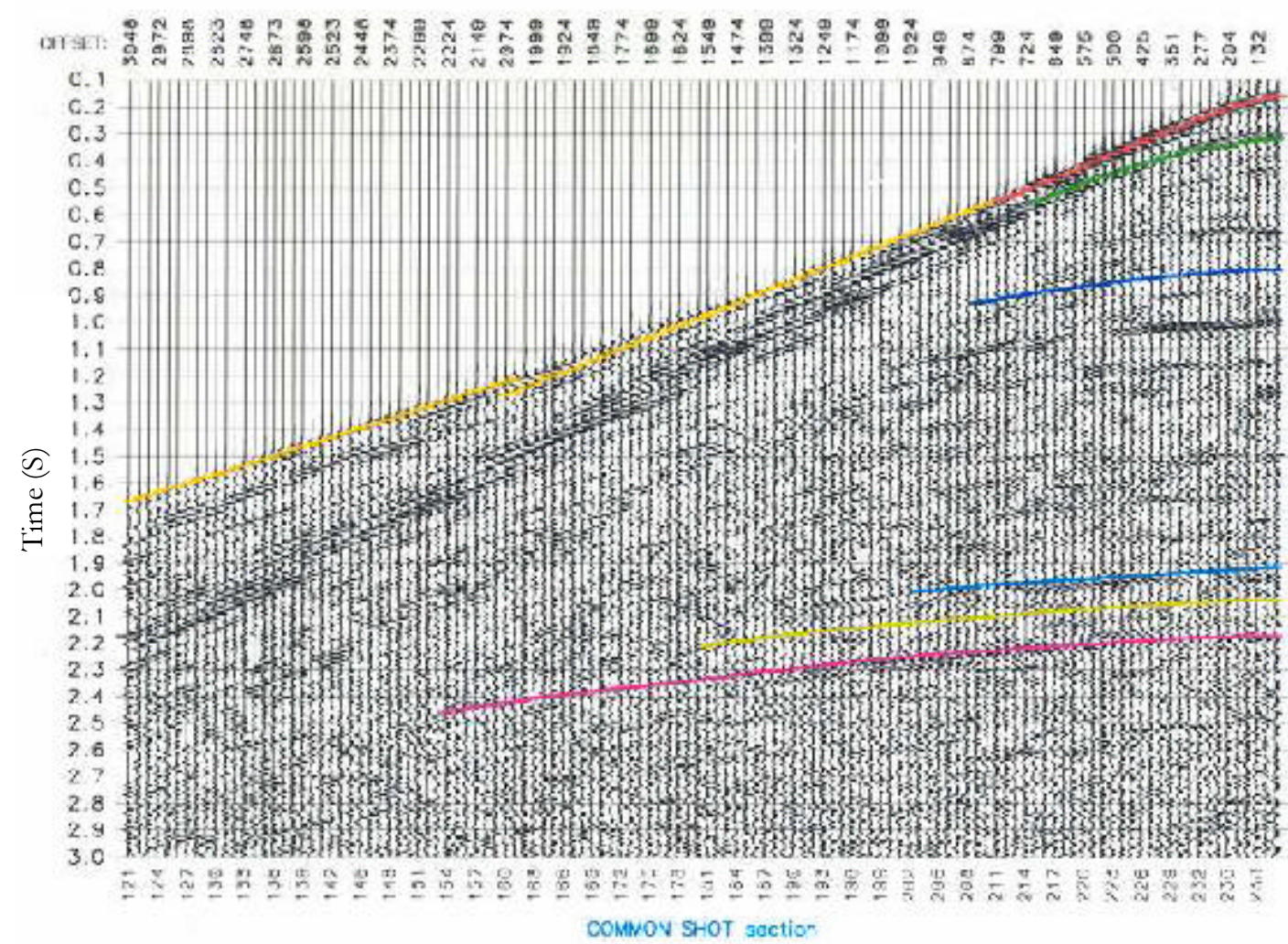

Fig. 6. Picked reflected/refracted events in common shot gather indicated by solid lines. 
A preliminary earth model having a few parameters was built and rays are traced through this model. The observed traveltimes are then compared with those obtained by a minimum time ray tracing and the residual traveltimes are subsequently converted into depth into reflection and refraction points. The refraction point is taken as the mid-point of the refracted wave along the interface. The velocity and interface depth are continuously updated by imposing that the dispersion of the reflected and refracted points be minimum (Figure 7) at which point the inversion process is stopped. Figures 8 and 9 show the models for the velocity field and interface depths in both 3-D and 2-D spaces.

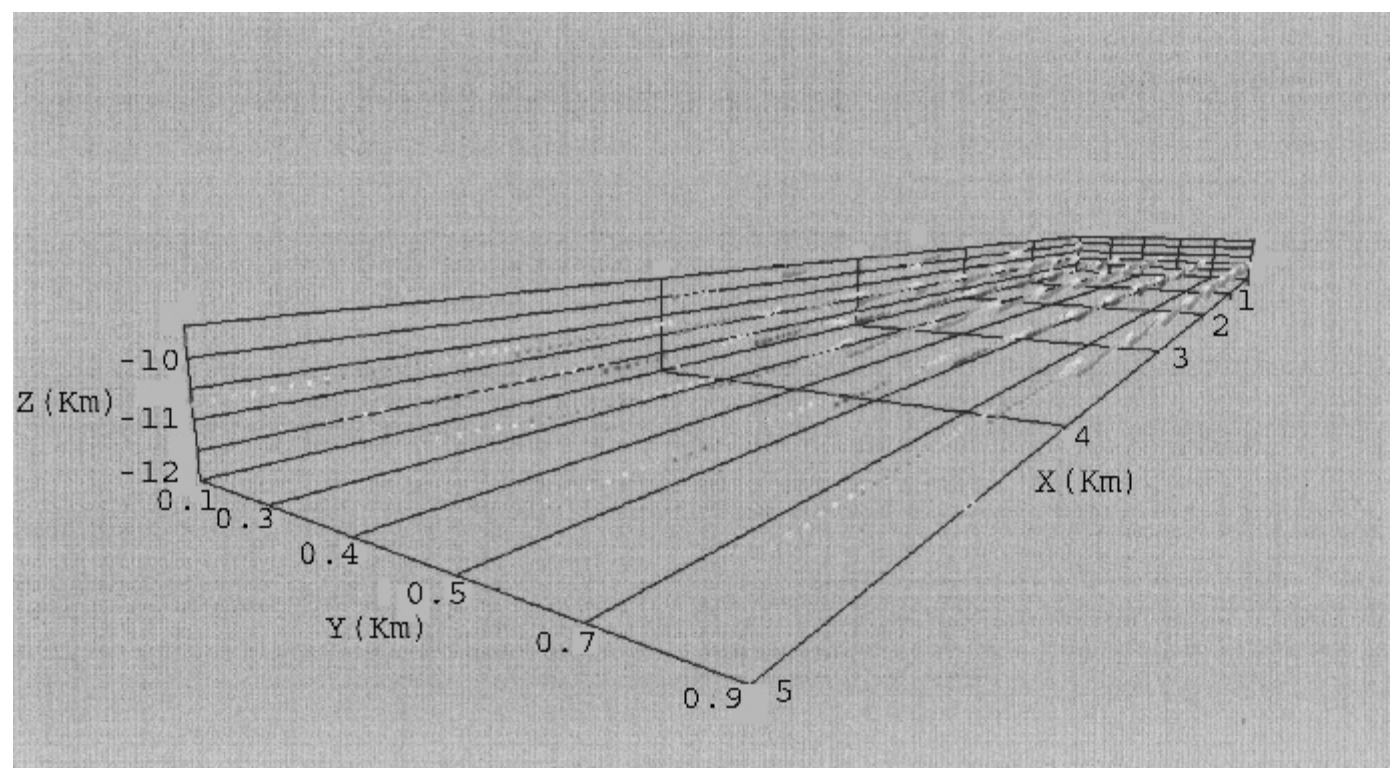

Fig. 7. Dispersion of points at an interface for both reflected and refracted waves along all the profiles.
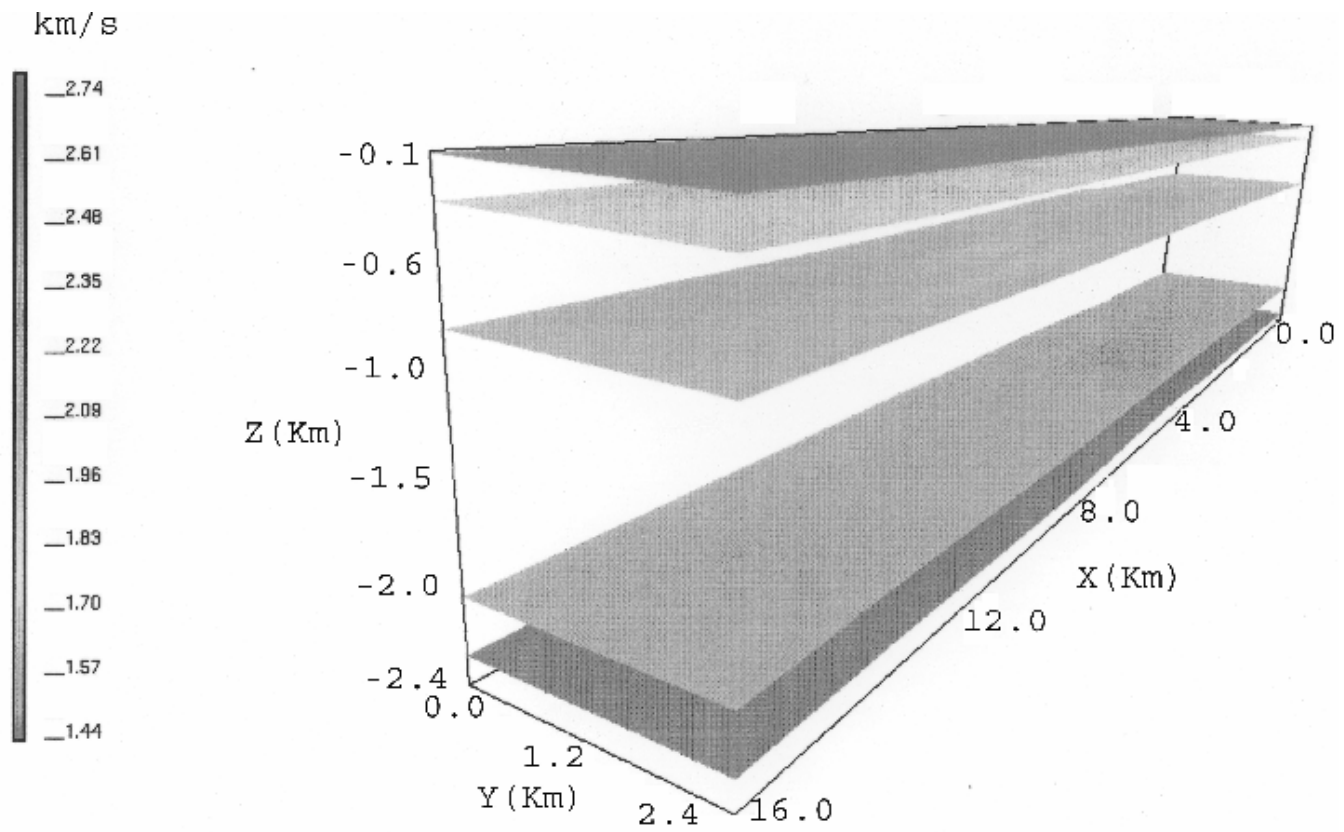

Fig. 8. Earth model obtained from 3-D tomographic inversion of both reflected and refracted waves. The velocities are in $\mathrm{km} / \mathrm{s}$. 


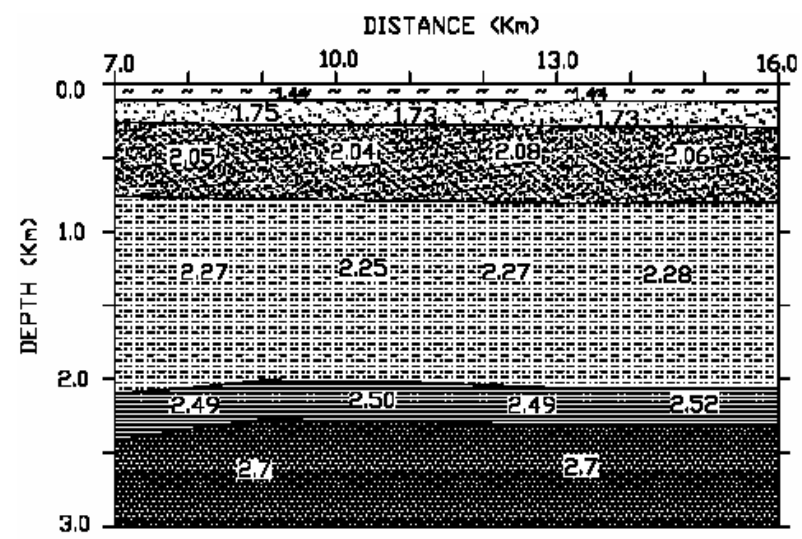

Fig. 9. 2-D earth model obtained from tomographic inversion of both reflected and refracted waves. The velocities are in $\mathrm{km} / \mathrm{s}$.

\section{TOMOGRAPHY AND MIGRATION}

Traveltime tomography can only give the velocity and depth imaging of geological structures associated with the picked events only. The events that are not picked are not processed by tomography. It is obvious that weak events as well as primary events masked by multiples and diffractions are difficult to pick. Moreover, deeper events due to low signal-to-noise ratio and incomplete coverage are difficult to isolate and follow all along the survey line. These and other problems put a restriction on the full use of the potential of the tomographic approach.

It is shown here that pre-stack depth migration is an important tool to offset the above difficulties and extract more information from the seismic data. Pre-stack depth migration has the capability of handling complex geologic structures and improving the detectability of weak reflected events. It also removes the diffractions that mask the weak events.

Pre-stack depth migration also serves as a litmus test of the correctness of the velocity field obtained by tomography. If the degree of coherence and continuity of the horizons can be ascertained from the depth migrated results one then concludes that the velocity model obtained by tomography is accurate.

Figure 10 shows a 2-D pre-stack depth migration based on the Kirchhoff method utilizing the velocity field obtained from the simultaneous tomographic inversion of reflected and refracted waves. All the fine details and weak reflected events can be seen clearly. The water bottom at a depth of $0.11 \mathrm{~km}$ is almost flat throughout the survey line.

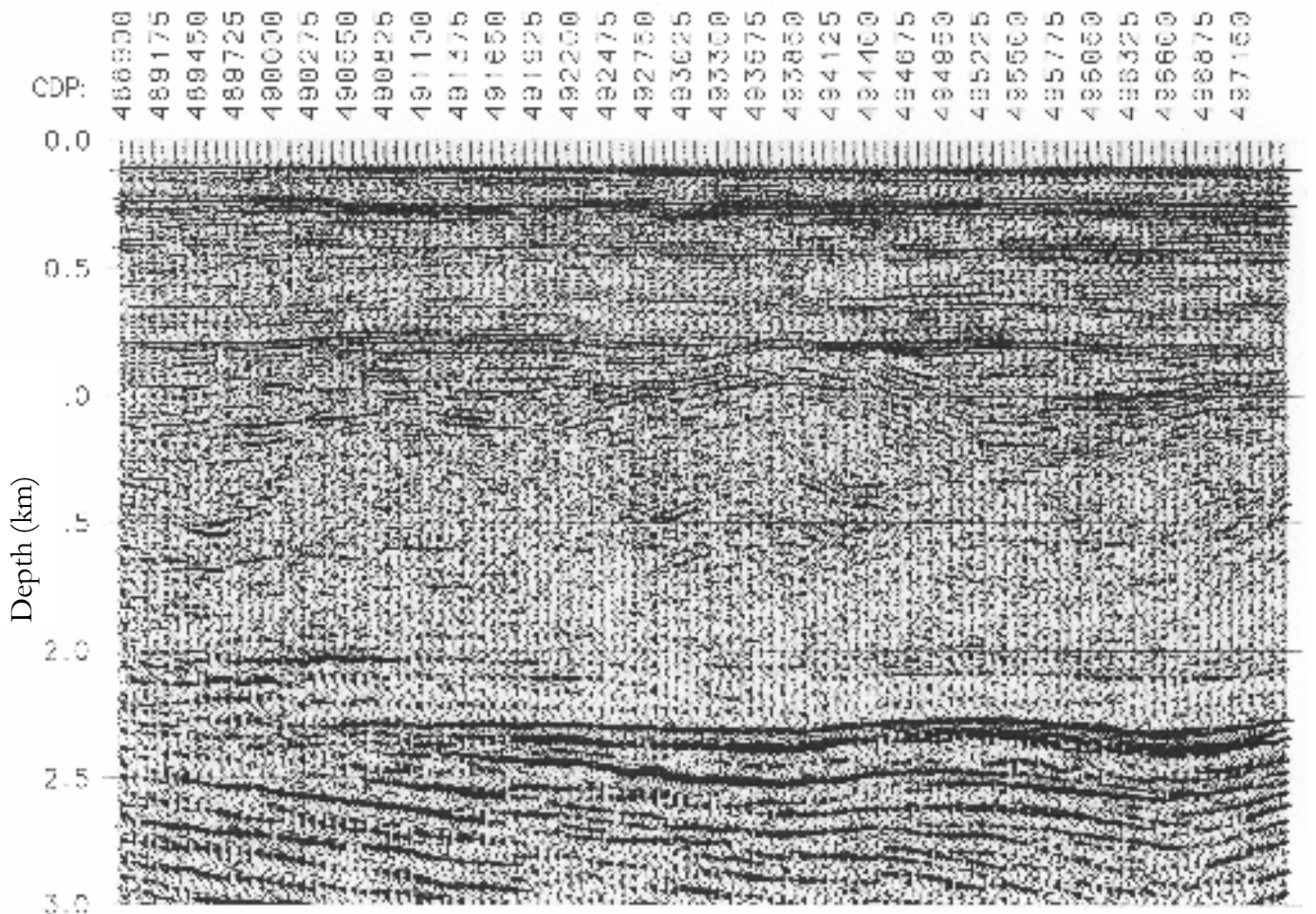

Fig. 10. Pre-stack depth migration utilizing the velocity field from tomographic inversion of both reflected and refracted waves. 
An interesting thing happens when the inversion result obtained by reflection tomography alone is compared with that obtained by joint inversion of reflected and refracted arrivals (Figure 11).

In Figure 11a where reflection alone is used a multiple is mapped at about a depth of $0.37 \mathrm{~km}$ throughout the survey line. Reverberations can also be seen at various depths. This is due to errors introduced during the picking phase and propagated in the final result. Because of the interference among primary and multiple reflections it is highly likely that a multiple is usually picked taken for a primary event. When refracted waves are included in the inversion process (Figure 11b) a controlling mechanism is introduced and errors in the picking of events are highly minimized. Refracted waves are the first to arrive at the receivers and do not suffer interferences from other wave types and therefore are clearly identified. Note that the multiple is almost removed in the joint inversion. The horizontal events at a depth of about $0.75 \mathrm{~km}$ are also better resolved. The blurring effect on a geologic structure in the middle of the section at about $1 \mathrm{~km}$ depth that is due to incomplete angular coverage is evident in Figure 11a. That effect is removed and clear anticlinal structure is obtained in the joint inversion. Comparison of the results in these figures with that of Figure 5 brings out a clear evidence of the superiority of the simultaneous tomographic inversion to that of the zero-offset stacked section.

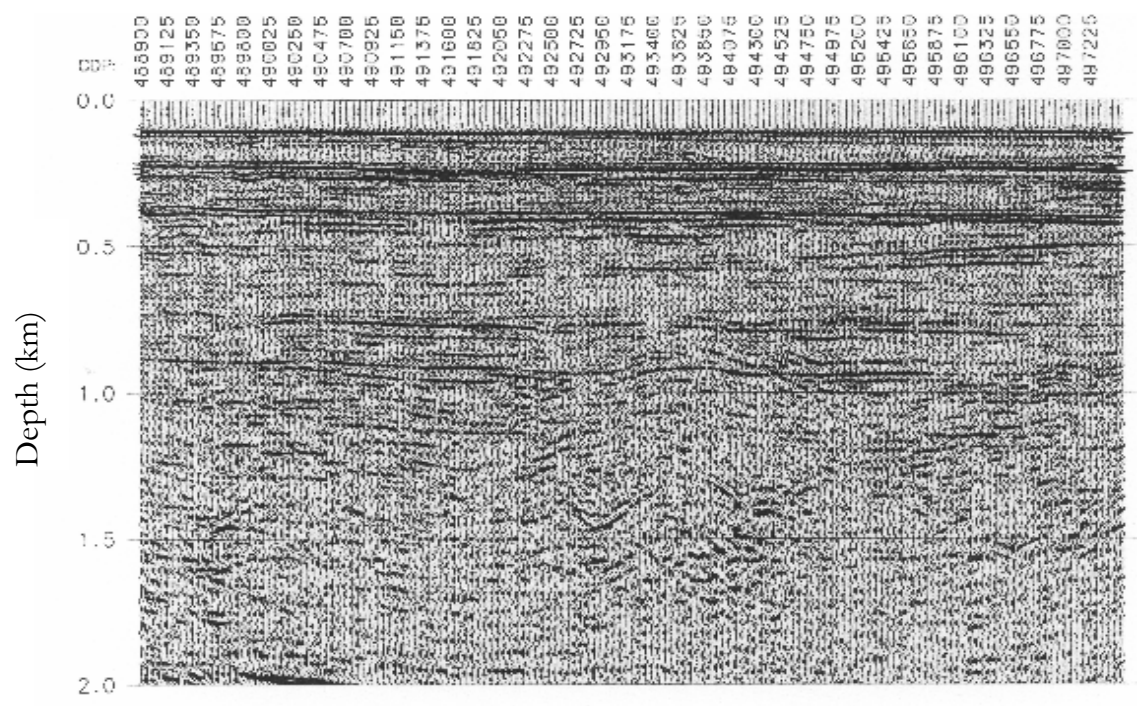

a

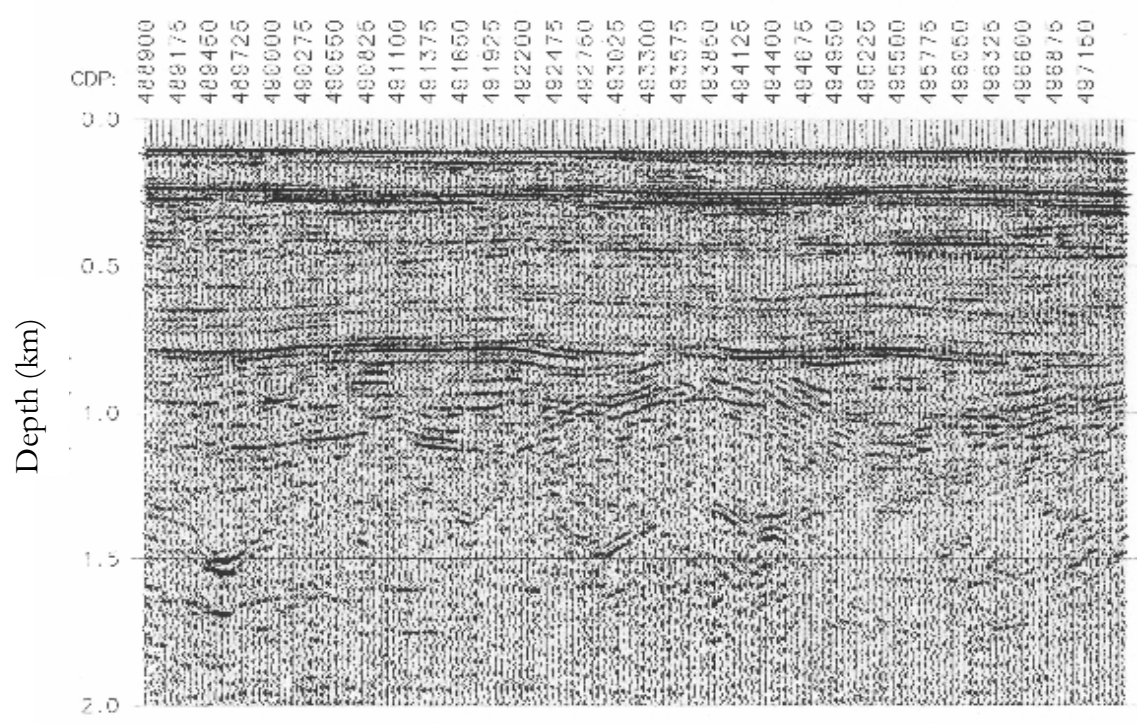

Fig. 11. Comparison of the pre-stack depth migrated image using velocity field obtained from tomographic inversion of reflected waves alone (a) and both reflected and refracted waves (b). 


\section{CONCLUSION}

The simultaneous tomographic inversion of reflection and refraction seismic data has produced a reliable and stable estimate of the velocity field and interface depth of the selected picked events that can be clearly identified in the common offset section or in the common shot gather. As short and long offset data are included in the inversion almost all the necessary wavelengths of the velocity spectrum are recovered. Subsequent prestack depth migration using the velocity model obtained from the tomographic inversion has given a complete picture of the whole data including those events that are weak and that could not be distinguished in the unmigrated sections. The use of pre-stack data has to some degree compensated for the incompleteness of the angular coverage.

It is also shown the superiority of the joint reflection and refraction tomography to reflection tomography alone. The blurring effects as well as error propagation due to mispicks in the reflection tomography are removed in the joint tomography. With the joint inversion the structural image and velocities of the derived model are significantly improved.

Pre-stack data with short and long offset reflection and refraction arrivals are capable of resolving the velocity-depth ambiguity and give a unique earth model without any blurring effect.

\section{ACKNOWLEDGMENTS}

This research was performed during my visit at OGS as a participant in the TRIL program at the Abdus Salam International Centre for Theoretical Physics (ICTP) in Trieste, Italy. I owe many thanks to Prof. G. Furlan, Director of TRIL for his continuous support. I am very much indebted to Aldo Vesnaver and his group at OGS for making my stay pleasant.

\section{REFERENCES}

1. Berryman, J.G. (1990). Stable iterative reconstruction algorithm for nonlinear traveltime tomography. Inverse Problems 6:21-42.

2. Bickel, S.H. (1990). Velocity-Depth ambiguity of reflection traveltimes. Geophysics 55:266-276.
3. Bohm, G., Carrion, P., Marchetti, A., Pettenati, F. and Vesnaver, A. (1993). Reflection tomography in complex structures. EAEG - 55 $5^{\text {th }}$ Meeting and Technical Exhibition, June 7-11. Stavanger, Norway.

4. Calnan, C. and Schuster, G. T. (1989). Reflection + transmission cross-well tomography. Expanded Abstracts. 59 ${ }^{\text {th }}$ Annual Int. SEG meeting, Dallas, USA.

5. Carrion, P.M. (1987). Inverse Problems and tomography in acoustics and Seismology. Penn. Publ. Co., Atlanta, GA.

6. Carrion, P.M. and Kuo, T. (1984). A method for computation of velocity profiles by inversion of large-offset records. Geophysics 49:1249-1258.

7. Carrion, P.M., Costa, J., Ferrer Pinheiro, J.E. and Schoenberg, M. (1992). Cross-hole tomography in anisotropic media. Geophysics 57:1194-1198.

8. Carrion, P.M., Boehm, G., Marchetti, A., Pettenati, F. and Vesnaver, A. (1993). Reconstruction of lateral gradients from reflection tomography. Journal of Seismic Exploration 2:55-67.

9. Johnstad, S.E., Uden, R.C. and Dunlop, K.N.B. (1993). Seismic reservoir monitoring over the Oseberg field. First Break 11:177-185.

10. Johnstad, S.E., Seymour, R.H. and Smith, P.J. (1995). Seismic reservoir monitoring over the Oseberg field during the period 1989-1992. First Break 13:169-183.

11. Lines, L. (1993). Ambiguity in analysis of velocity and depth. Geophysics 58:596-597.

12. Mao, W. and Stuart, G.W. (1997). Transmission reflection tomography: Application to reverse VSP data. Geophysics 62:884-894.

13. Ross, W.S. (1994). The velocity-depth ambiguity in seismic traveltime data. Geophysics 59:830-843.

14. Schuster, G. T. (1989). Analytic generalized inverse for transmission + reflection tomography. Geophysics 54:1046-1049.

15. Stork, C. and Clayton, R.W. (1991). Linear aspects of tomographic velocity analysis. Geophysics 56:483-495.

16. Stork, C. (1992). Singular value decomposition of the velocity-reflector depth tradeoff, Part 2: Highresolution analysis of a generic model. Geophysics 57:933-943.

17. Tieman, H.J. (1994). Investigating the velocity-depth ambiguity of reflection traveltimes. Geophysics 59:1763-1773.

18. White, D.J. (1989). Two-dimensional seismic refraction tomography. Geophysical Journal 97:223-245. 